\title{
Leishmania donovani-Induced Immune Dysregulation among Sudanese Patients with Visceral and Post Kala-Azar Dermal Leishmaniases: Possible Roles in Pathogenesis
}

\author{
Elsamani Elmotwafig Mohamed Elsammani1", Brima Younis Musa1, Mona Elfaki Eltahir Elfaki², \\ Ahmed Mudawi Mudawi1 , Eltahir Awad Gasim Khalil1* (1) \\ ${ }^{1}$ Institute of Endemic Diseases, University of Khartoum, Khartoum, Sudan \\ ${ }^{2}$ Department of Microbiology and Clinical Parasitology, College of Medicine, King Khalid University, Abha, KSA \\ Email: ^eltahirk@iend.org
}

How to cite this paper: Elsammani, E.E.M., Musa, B.Y., Elfaki, M.E.E., Mudawi, A.M. and Khalil, E.A.G. (2020) Leishmania donovani-Induced Immune Dysregulation among Sudanese Patients with Visceral and Post Kala-Azar Dermal Leishmaniases: Possible Roles in Pathogenesis. Advances in Infectious Diseases, 10, 235-242.

https://doi.org/10.4236/aid.2020.105021

Received: October 20, 2020

Accepted: November 22, 2020

Published: November 25, 2020

Copyright $\odot 2020$ by author(s) and Scientific Research Publishing Inc. This work is licensed under the Creative Commons Attribution International License (CC BY 4.0).

http://creativecommons.org/licenses/by/4.0/

\begin{abstract}
L. donovani infections (visceral and post kala-azar dermal leishmaniases) are characterized by infection-induced reversible immune suppression. Autoimmunity is a well-documented phenomenon among patients with primary immune deficiencies. This study aimed to study auto-immune phenomena accompanying $L$. donovani infections. In a prospective case-controlled study and following informed consent, 155 individuals with visceral leishmaniasis (VL; $n=62$ ), post kala-azar dermal leishmaniasis (PKDL; $n=31$ ) and apparently healthy volunteers $(n=62)$ were recruited. Sera antinuclear (ANA), anti-dsDNA, anti-thyroid peroxidase (TPO), anti-smooth muscles (ASMA) and F-actin auto-antibodies were measured using ELISA and indirect immune-fluorescence assay. The mean ages of VL, PDKL patients and apparently healthy volunteers were: $17.5 \pm 12.5,15.0 \pm 7.0$ and $17.5 \pm 9.5$ years with Male:Female ratios of 2:0, 1:2 and 1:5 respectively. Significantly high frequencies of F-actin $(74.2 \% ; 46 / 62)$ and ASMA $(50 \% ; 31 / 62)$ auto-antibodies were seen among VL patients $(p=0.003, p=0.001)$ compared to apparently healthy volunteers. Likewise, significantly high frequencies of F-actin (64.5\%; $20 / 31 ; \boldsymbol{p}=0.001)$, ASMA $(42 \% ; 13 / 31 ; \boldsymbol{p}=0.003)$, ANA $(36 \% ; 11 / 31 ; \boldsymbol{p}=0.001)$ and anti-dsDNA $(16 \% ; 5 / 31 ; p=0.01)$ auto-antibodies were seen among PKDL patients. Development of tissue-based autoantibodies in L. donovani infections probably indicates loss of peripheral tolerance with activation of circulating auto-reactive $\mathrm{T}$ and $\mathrm{B}$ cells probably contributing to disease pathogenesis (increased bilirubin/liver enzymes, prolonged QT interval/arrythmias and blood cytopenias). In conclusion, $L$. donovani infection-induced immune
\end{abstract}


suppression with development of tissue-based auto-antibodies is prevalent among Sudanese patients with VL and PKDL leishmaniases and contributes to some aspects of the disease pathogenesis.

\section{Keywords}

L. donovani-Induced Immune Suppression, Tissue-Based Autoimmunity, Pathogenesis

\section{Introduction}

The leishmaniases are increasingly recognized major public health problems that constitute wide spectra of human and animal diseases. It is caused by obligate intra uni-cellular protozoan parasites of the genus Leishmania. Clinical manifestations depend on complex interactions between the Leishmania parasite and human host immune system. Visceral leishmaniasis (VL, Kala-azar) is the most severe form with high mortality rates if untreated. Control of VL focuses on case detection, drug treatment, control of animal reservoir/vectors and bed nets. Research on anti-leishmania candidate vaccines is on-going [1]-[6]. More than fifty per cent of successfully treated VL patients develop an immune-mediated dermatosis called post kala-azar dermatitis (PKDL). Early activation of the innate immune system takes place during $L$. donovani infection enabling the host immune system to determine the type of immune response (Th1/Th2). The majority of VL patients become immune-compromised with depressed cell-mediated immunity and anergy to some antigens (leishmanial and purified protein derivative). VL-induced immune suppression is transient with reversion to immune competence after six months of successful treatment. VL-immune deficiency is probably due to on-site generation of adenosine, a potent immune-suppressant that leads to immune impairment and disease progression. L. donovani infection-induced auto-immune phenomena probably happen when auto-reactive $B$ and $\mathrm{T}$ cells escape tolerance check points with resultant hyper-gamma-globulinemia and immune-related peripheral blood cytopenias. Alternatively, the infection leads to T-cells suppression with enhanced leishmanial antigen-specific IL-10 production and increased CD8+ CD28-mediated T CD4+ cells anergy. The mechanisms of hyper-gamma-globulinemia that accompanies VL and PKDL are probably similar to what happens in Hyper Ig $M$ syndrome (a primary immune deficiency syndrome [2] [4] [7]-[22]). This study aimed to characterize auto-immunity (organ-specific and tissue-specific auto-antibodies) that accompanies $L$. donovani infection-induced immune suppression. Autoimmunity is generally induced by genetics and environmental factors (microbial infections etc.). A number of theories have been proposed to explain autoimmune phenomena in PID: failure of tolerance mechanisms with failure of deletion of autoreactive $B$ cells), signaling pathway defects with failure of anergy, loss of development of $\mathrm{T}_{\text {reg }}$ cells, deranged $\mathrm{T}$ cell activation by innate system and defects in self-antigen 
clearance that follows tissue destruction. Autoantibodies (anti-ANA, anti-ds DNA, anti-RNP, anti-SS/A, anti-SS/B, anti-cardiolipin, anti-beta 2-glycoprotein I, anti-actin, anti-tubulin, anti-smooth muscle) have been detected in the sera of animals and humans with cutaneous and visceral leishmaniases [23]-[34]. Although a pathogenic role for autoimmunity in leishmaniasis was reported (immune pancytopenia), it has to be clearly understood that reactivity to one or more autoantibodies should not be mis-interpreted as autoimmune disorders. In addition, a number of autoimmune diseases (SLE, rheumatoid arthritis, polyarteritis nodosa and autoimmune hemolytic anemia, autoimmune hepatitis) could be mimicked or exacerbated by leishmaniasis [29] [35]-[40]. This study aimed to determine the emergence of auto-immunity following secondary immune suppression caused by $L$. donovani infections.

\section{Materials and Methods}

\subsection{Study Type and Site}

This was a prospective and case-controlled study with a case: control ratio of 1:1 - 1:2. The study proposal was reviewed and passed by the Scientific and Ethics Committees of the Institute of Endemic Diseases; University of Khartoum. Written informed consents were obtained from participating individuals. Visceral and PKDL patients were recruited at Professor Elhassan Centre, Dooka, Eastern Sudan (VL endemic area). Apparently Healthy controls were recruited in Greater Khartoum, Khartoum (VL non-endemic area).

\subsection{Study Patients and Apparently Healthy Controls}

One hundred and fifty five VL $(\mathrm{n}=62)$, PKDL $(\mathrm{n}=31)$ parasitologically-confirmed patients and apparently healthy volunteers $(\mathrm{n}=62)$ were enrolled. The sample size was calculated using EpiInfo version 3.04.04 software. Taking into consideration that the proportion of VL patients who will develop PKDL is 50\% (0.5), two-sided significant level as 0.05 and a power of $80 \%$ and the ratio of the sample size of exposed/non-exposed $=1$. The total sample size was calculated at 155 [Odds ratio $=3.5$ and prevalence ratio $=1.75$ ]. VL/PKDL patients and volunteers were recruited sequentially from patients/individuals of all ages from both sexes who report to with HBV, HCV, HIV and chronic diseases (Diabetes mellitus, chronic renal failure etc.), pregnant and lactating females were excluded.

\subsection{Methods}

Anti-ANA (anti-nuclear antibody), anti-ds-DNA and anti-TPO auto-antibodies were measured in sera by indirect ELISA using commercial ELISA kits as described by the manufacture (EUROIMMUN Medizinische Labor Diagnostika AG, Germany). ASMA and F-actin auto-antibodies were measured in serum by indirect immune-fluorescence technique using commercial Immune-fluorescence kits as described by the manufacture (EUROIMMUN, Medizinische Labor Diagnostika AG, Germany). 


\subsection{Statistical Analysis}

The data were analysed using Epidemiological Information Software (Epi Info 7). The frequencies of auto-antibodies (ANA, ds-DNA, TPO, ASMA and F-actin) were calculated. The frequencies of auto-antibodies detected were compared between the VL group and apparently healthy volunteers group; PKDL group and apparently healthy volunteers group and between PKDL and VL groups. P value $<0.05$ was considered statistically significant.

\section{Results}

The means ages and the male; female ratios of the study groups were: VL patients $(n=62$, mean \pm SD age $=17.5 \pm 12.5$ and a Male: Female ratio of 1.2:1), PKDL patients $(\mathrm{n}=31$, mean \pm SD age $=15 \pm 7$ years and a Male: Female ratio of 2:1) and apparently healthy volunteers from VL non-endemic area $(\mathrm{n}=62$, mean \pm SD age, $27.5 \pm 9.5$ years; and a Male: Female ratio of 1.5:1). Significantly high frequencies of F-actin $(74.2 \%, 46 / 62 ; \boldsymbol{p}=\mathbf{0 . 0 0 3})$ and ASMA (50\%, 31/62; $\boldsymbol{p}$ $=0.001$ ) auto-antibodies were reported among VL patients compared to apparently healthy volunteers. Likewise, significantly high frequencies of F-actin

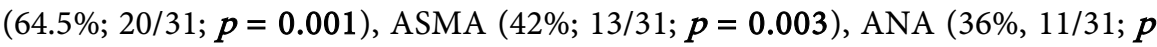
$=0.001)$ and anti-dsDNA $(16 \%, 5 / 31 ; p=0.01)$ auto-antibodies were reported among PKDL patients. Eleven apparently healthy volunteers $(11 / 62,17.7 \%)$ in total had one or more auto-antibodies (Table 1).

\section{Discussion}

Autoimmunity in different forms has been described intensively in patients with primary immune deficiency (PID) several explanations have been put forward to explain this ironic discrepancy. The mechanisms of autoimmunity during $L$. donovani infection and the clinical significance of this autoimmunity among Sudanese VL/PKDL patients are not clear and remain to be determined, but

Table 1. Frequencies of auto-antibodies among different study groups.

\begin{tabular}{cccccc}
\hline Study Group & ANA $^{*}$ & Anti-dsDNA & ASMA & F-actin & anti-TPO \\
\hline VL patients & $1 / 62$ & $0 / 62$ & $31 / 62$ & $46 / 62$ & $0 / 62$ \\
$(n=62)$ & $(1.6 \%)$ & $(0 \%)$ & $(50 \%)$ & $(74.2 \%)$ & $(0 \%)$ \\
$p=$ & 0.5 & 0.5 & 0.00000003 & 0.0000001 & - \\
PKDL patients & $11 / 31$ & $5 / 31$ & $13 / 31$ & $20 / 31$ & $0 / 31$ \\
$(n=31)$ & $35 \%$ & $16 \%$ & $42 \%$ & $64.5 \%$ & - \\
$p=$ & 0.0000001 & 0.02 & 0.00000003 & 0.00000001 & - \\
Apparently Healthy & $2 / 62$ & $1 / 62$ & $4 / 62$ & $4 / 62$ & $0 / 62$ \\
$(n=62)$ & $(3.3 \%)$ & $(1.7 \%)$ & $(6.6 \%)$ & $(6.6 \%)$ & -
\end{tabular}

${ }^{*} \mathrm{ANA}=$ antinuclear antibody. 
appear to be similar to that accompany PID. Development of tissue-based auto-antibodies in $L$. donovani infections as was commonly seen among our $\mathrm{VL} / \mathrm{PKDL}$ patients probably indicates absence of peripheral tolerance mechanisms contributing to activation of autoreactive $\mathrm{T}$ and $\mathrm{B}$ cells. The clinical significance of these differences remains unclear, but the development of autoimmunity can explain some pathogenetic features of $L$. donovani infection, especially pre-treatment prolonged QT interval, cardiac arrhythmias, increased serum bilirubin and liver enzymes. These abnormalities could indicate infection-induced heart and liver involvement and might explain sudden deaths encountered during antimony treatment. It is our assumption that the presence of high titers of smooth-muscle auto antibodies (ASMA) with significant reactivity against antinuclear and filamentous actin (F-actin) in VL/PKDL patients, could explain cardiac and liver involvement in VL/PKDL patients. The presence of F-actin has been recognized as specific to auto immune hepatitis (AIH) [37].

\section{Conclusion}

In conclusion, tissue-specific auto-antibodies (ASMA, F-actin) are prevalent among Sudanese visceral and post kala-azar dermal leishmaniasis patients and could explain aspects of the disease pathogenesis.

\section{Declaration}

This work is a result original work conducted as part of research study that was conducted at treatments firm of the Institute of Endemic Diseases, University of Khartoum, Sudan. The authors declare that this work is not under consideration for publication by any publishing firm.

\section{Funding}

This study received no financial support from any source.

\section{Authors' Contribution}

Conceptualization, EEEM and EAGK; data curation, EEEM, MEEE; methodology, BYM, AMM and MEEE; data analysis, EEEM, MEEE, EAGK; writing-original draft, EEEM, MEEE, EAGK; writing-review \& editing, EEEM, MEEE, EAGK. All authors approved the final version of the manuscript.

\section{Conflicts of Interest}

The authors declare no conflicts of interest regarding the publication of this paper.

\section{References}

[1] Kumar, R. and Nylén, S. (2012) Immunobiology of Visceral Leishmaniasis. Frontiers in Immunology, 3, 251. https://doi.org/10.3389/fimmu.2012.00251

[2] Zijlstra, E.E. (2001) Leishmaniasis in Sudan Leishmaniasis. Transactions of the 
Royal Society of Tropical Medicine and Hygiene, 89, 647-652.

[3] Kamil, A.A., Khalil, E.A.G., Musa, A.M., et al. (2003) Alum-Precipitated Autoclaved Leishmania Major plus Bacille Calmette-Guérrin, a Candidate Vaccine for Visceral Leishmaniasis: Safety, Skin-Delayed Type Hypersensitivity Response and Dose Finding in Healthy Volunteers. Transactions of the Royal Society of Tropical Medicine and Hygiene, 97, 365-368. https://doi.org/10.1016/S0035-9203(03)90171-4

[4] El Hassan, A.M. and Khalil, E.A. (2001) Post-Kala-Azar Dermal Leishmaniasis: Does It Play a Role in the Transmission of Leishmania donovani in the Sudan? Tropical Medicine \& International Health, 6, 743-744. https://doi.org/10.1046/j.1365-3156.2001.00776.x

[5] Khalil, E.A.G., Ayed, N.B., Musa, A.M., et al. (2005) Dichotomy of Protective Cellular Immune Responses to Human Visceral Leishmaniasis. Clinical \& Experimental Immunology, 140, 349-353. https://doi.org/10.1111/j.1365-2249.2005.02768.x

[6] Alvar, J., Vélez, I.D., Bern, C., Herrero, M., Desjeux, P., Cano, J., Jannin, J. and de Boer, M. (2012) Leishmaniasis Worldwide and Global Estimates of Its Incidence. PLoS ONE, 7, e35671. https://doi.org/10.1371/journal.pone.0035671

[7] Zijlstra, E.E., El-Hassan, A.M. and Zijlstra, E.E. (1993) Leishmanin and Tuberculin Sensitivity in Leishmaniasis in the Sudan, with Special Reference to Kala-Azar. Transactions of the Royal Society of Tropical Medicine and Hygiene, 87, 425-427. https://doi.org/10.1016/0035-9203(93)90024-K

[8] Zijlstra, E.E., Musa, A.M., Khalil, E.A.G., El Hassan, I.M. and El-Hassan, A.M. (2003) Post-Kala-Azar Dermal Leishmaniasis. Lancet Infectious Diseases, 3, 87-98. https://doi.org/10.1016/S1473-3099(03)00517-6

[9] Ismail, A., Khalil, E.A.G., Musa, A.M., El Hassan, I.M., Ibrahim, M.E., Theander, T.G. and El Hassan, A.M. (2006) The Pathogenesis of Post Kala-Azar Dermal Leishmaniasis from the Field to the Molecule: Does Ultraviolet Light (UVB) Radiation Play a Role? Medical Hypotheses, 66, 993-999.

https://doi.org/10.1016/j.mehy.2005.03.035

[10] Ansari, N.A., Kumar, R., Raj, A. and Salotra, P. (2008) Elevated Levels of IgG3 and IgG4 Subclass in Paediatric Cases of Kala Azar. Parasite Immunology, 30, 403-409. https://doi.org/10.1111/j.1365-3024.2008.01036.x

[11] Mohamed-Ahmed, A.H.A., Brocchini, S. and Croft, S.L. (2012) Recent Advances in Development of Amphotericin B Formulations for the Treatment of Visceral Leishmaniasis. Current Opinion in Infectious Diseases, 25, 695-702. https://doi.org/10.1097/QCO.0b013e328359eff2

[12] Paletta-Silva, R. and Meyer-Fernandes, J.R. (2012) Adenosine and Immune Imbalance in Visceral Leishmaniasis: The Possible Role of Ectonucleotidases. Journal of Tropical Medicine, 2012, Article ID: 650874. https://doi.org/10.1155/2012/650874

[13] Faleiro, R.J., Kumar, R., Hafner, L.M. and Engwerda, C.R. (2014) Immune Regulation during Chronic Visceral Leishmaniasis. PLOS Neglected Tropical Diseases, 8 , e2914. https://doi.org/10.1371/journal.pntd.0002914

[14] Zijlstra, E.E. (2016) The Immunology of Post-Kala-Azar Dermal Leishmaniasis (PKDL). Parasites \& Vectors, 9, 464. https://doi.org/10.1186/s13071-016-1721-0

[15] Benoist, C. and Mathis, D. (2001) Autoimmunity Provoked by Infection: How Good Is the Case for T Cell Epitope Mimicry? Nature Immunology, 2, 797-801. https://doi.org/10.1038/ni0901-797

[16] Wucherpfennig, K.W. (2001) Mechanisms for the Induction of Autoimmunity by Infectious Agents. Journal of Clinical Investigation, 108, 1097-1104.

https://doi.org/10.1172/JCI200114235 
[17] Bussone, G. and Mouthon, L. (2009) Autoimmune Manifestations in Primary Immune Deficiencies. Autoimmunity Reviews, 8, 332-336. https://doi.org/10.1016/j.autrev.2008.11.004

[18] Jesus, A.A., Duarte, A.J.S. and Oliveira, J.B. (2008) Autoimmunity in Hyper-IgM Syndrome. Journal of Clinical Immunology, 28, S62-S66. https://doi.org/10.1007/s10875-008-9171-x

[19] Meffre, E. and Wardemann, H. (2008) B-Cell Tolerance Checkpoints in Health and Autoimmunity. Current Opinion in Immunology, 20, 632-638. https://doi.org/10.1016/j.coi.2008.09.001

[20] Kühne, V. and Büscher, P. (2019) The Unknown Nature of the Antigen in the Direct Agglutination Test for Visceral Leishmaniasis Hampers Development of Serodiagnostic Tests. American Journal of Tropical Medicine and Hygiene, 100, 246-255. https://doi.org/10.4269/ajtmh.18-0740

[21] Mukherjee, S., Sengupta, R., Mukhopadhyay, D., Braun, C., Mitra, S., Roy, S., et al. (2019) Impaired Activation of Lesional CD8+ T-Cells Is Associated with Enhanced Expression of Programmed Death-1 in Indian Post Kala-Azar Dermal Leishmaniasis. Scientific Reports, 9, Article No. 762. https://doi.org/10.1038/s41598-019-48640-0

[22] Zijlstra, E.E. (2019) Biomarkers in Post-Kala-Azar Dermal Leishmaniasis. Frontiers in Cellular and Infection Microbiology, 9, 228. https://doi.org/10.3389/fcimb.2019.00228

[23] Santiago, M., Martinelli, R., Ko, A., Reis, E.A., Fontes, R.D., Nascimento, E.G., et al. (2001) Anti- $\beta 2$ Glycoprotein I and Anticardiolipin Antibodies in Leptospirosis, Syphilis and Kala-Azar. Clinical and Experimental Rheumatology, 19, 425-430.

[24] Schurman, C. (2003) Autoimmunity in Wiskott-Aldrich Syndrome. Current Opinion in Rheumatology, 15, 446-453.

https://doi.org/10.1097/00002281-200307000-00012

[25] Gambineri, E., Torgerson, T.R. and Ochs, H.D. (2003) Immune Dysregulation, Polyendocrinopathy, Enteropathy, and X. Current Opinion in Rheumatology, 15, 430-435. https://doi.org/10.1097/00002281-200307000-00010

[26] Rioux, J.D. and Abbas, A.K. (2005) Paths to Understanding the Genetic Basis of Autoimmune Disease. Nature, 435, 584-589. https://doi.org/10.1038/nature03723

[27] Atta, A.M., Carvalho, E.M., Jerônimo, S.M.B. and Sousa Atta, M.L.B. (2007) Serum Markers of Rheumatoid Arthritis in Visceral Leishmaniasis: Rheumatoid Factor and Anti-Cyclic Citrullinated Peptide Antibody. Journal of Autoimmunity, 28, 55-58. https://doi.org/10.1016/j.jaut.2006.12.001

[28] Moraes-Vasconcelos, D., Costa-Carvalho, B.T., Torgerson, T.R. and Ochs, H.D. (2008) Primary Immune Deficiency Disorders Presenting as Autoimmune Diseases: IPEX and APECED. Journal of Clinical Immunology, 28, 11-19. https://doi.org/10.1007/s10875-008-9176-5

[29] Sakkas, L.I., Boulbou, M., Kyriakou, D., Makri, I., Sinani, C., Germenis, A. and Stathakis, N. (2008) Immunological Features of Visceral Leishmaniasis May Mimic Systemic Lupus Erythematosus. Clinical Biochemistry, 41, 65-68. https://doi.org/10.1016/j.clinbiochem.2007.10.008

[30] Chapel, H. and Cunningham-Rundles, C. (2009) Update in Understanding Common Variable Immunodeficiency Disorders (CVIDs) and the Management of Patients with These Conditions. British Journal of Haematology, 145, 709-727. https://doi.org/10.1111/j.1365-2141.2009.07669.x

[31] Münz, C., Lünemann, J.D., Getts, M.T. and Miller, S.D. (2009) Antiviral Immune 
Responses: Triggers of or Triggered by Autoimmunity? Nature Reviews Immunology, 9, 246-258. https://doi.org/10.1038/nri2527

[32] Cunningham-Rundles, C. (2001) Autoimmunity in Primary Immune Deficiency: Taking Lessons from Our Patients. Clinical \& Experimental Immunology, 164, 6-11. https://doi.org/10.1111/j.1365-2249.2011.04388.x

[33] Walker, L.S.K. and Abbas, A.K. (2002) The Enemy Within: Keeping Self-Reactive T Cells at Bay in the Periphery. Nature Reviews Immunology, 2, 11-19. https://doi.org/10.1038/nri701

[34] Granel, B., Serratrice, J., Swiader, L., Gambarelli, F., Daniel, L., Fossat, C., et al. (2000) Crossing of Antinuclear Antibodies and Anti-Leishmania Antibodies. Lupus, 9, 548-550. https://doi.org/10.1177/096120330000900713

[35] Voulgari, P.V., Pappas, G.A., Liberopoulos, E.N., Elisaf, M., Skopouli, F.N. and Drosos, A.A. (2004) Visceral Leishmaniasis Resembling Systemic Lupus Erythematosus. Annals of the Rheumatic Diseases, 63, 1348-1349. https://doi.org/10.1136/ard.2003.014480

[36] Erduran, E., Bahadir, A. and Gedik, Y. (2005) Kala-Azar Associated with Coombs-Positive Autoimmune Hemolytic Anemia in the Patients Coming from the Endemic Area of This Disease and Successful Treatment of These Patients with Liposomal Amphotericin B. Pediatric Hematology and Oncology, 22, 349-355. https://doi.org/10.1080/08880010590964110

[37] Dalgiç, B., Dursun, I. and Akyol, G. (2005) A Case of Visceral Leishmaniasis Misdiagnosed as Autoimmune Hepatitis. Turkish Journal of Gastroenterology, 16, 52-53.

[38] Makaritsis, K.P., Gatselis, N.K., Ioannou, M., Petinaki, E. and Dalekos, G.N. (2009) Polyclonal Hypergammaglobulinemia and High Smooth-Muscle Autoantibody Titers with Specificity against Filamentous Actin: Consider Visceral Leishmaniasis, Not Just Autoimmune Hepatitis. International Journal of Infectious Diseases, 13, e157-e160. https://doi.org/10.1016/j.ijid.2008.08.011

[39] Werneck, G.L., Batista, M.S.A., Gomes, J.R.B., Costa, D.L. and Costa, C.H.N. (2003) Prognostic Factors for Death from Visceral Leishmaniasis in Teresina, Brazil. Infect, 31, 174-177. https://doi.org/10.1007/s15010-003-3139-9

[40] Madalosso, G., Fortaleza, C.M., Ribeiro, A.F., Cruz, L.L., Nogueira, P.A. and Lindoso, J.A.L. (2012) American Visceral Leishmaniasis: Factors Associated with Lethality in the State of São Paulo, Brazil. Journal of Tropical Medicine, 2012, Article ID: 281572. https://doi.org/10.1155/2012/281572 